\title{
A robust controller for a variable-speed wind turbine using a single mass model
}

\author{
Aazdine AFKARI*, Fatima Ez-zahra LAMZOURI, El-Mahjoub BOUFOUNAS
}

REIPT Laboratory, Faculty of Sciences and Technology, Moulay Ismail University of Meknes, BP 509, Boutalamine 52000, Errachidia, Morocco

\begin{abstract}
In this paper, a robust nonlinear controller based on integral sliding mode control (ISMC) strategy was proposed to maximize the captured energy for a variable speed wind turbine (VSWT). In order to reduce the reaching phase, an integral action is introduced in the switching surface of the sliding mode control (SMC) for a minimum steady state error. The controlled system stability is demonstrated using the Lyapunov theory and the trajectory tracking error converges in finite time to zero without any chattering problems. Various simulations, carried out via Matlab software, are discussed to highlight the proposed method performance, using a nonlinear single mass model. Finally, the simulation results show that the proposed Integral SMC strategy ensures better response speed and smaller steady-state error compared to the standard SMC.
\end{abstract}

*Corresponding author: azdine.afkari@gmail.com 


\section{Introduction}

Wind energy is an abundant renewable source of new electrical generation capacity in the world, and it is exploited by converting the kinetic energy of moving air mass into electricity; therefore, it is necessary to introduce tools to make the wind turbine (WT) more profitable [1]. The variable speed horizontal axis wind turbine is mainly considered in this study, due to its great ability in the extraction of energy [2]. However, variable speed system is more complex and requires an efficient control strategy [3]. Various works have been reported to the control of the mechanical as well as the electrical components. This work is intended to the mechanical part, with the main objective, below the nominal power, is to maximize the energy captured from the wind and to minimize the mechanical stress in the system that takes into consideration the nonlinear nature of the system behavior and the flexibility of the drive-train shaft.

The sliding mode control (SMC) [4-6], is widely used in many applications, especially, the control of a variable speed wind turbine (VSWT). This is due to its property of robustness with respect to uncertainties and disturbances [7,8]. The chattering behavior is especially the main problem in the design of SMC. One possible method to solve this problem is the boundary layer approach [8]. This technique has given good results when the system uncertainties are small. However, when these uncertainties are large, a high gain is needed and higher amplitude of chattering is produced. The integral sliding mode control (ISMC) [9-11], is a robust control for improving the performance of standard sliding mode control. It is to design a sliding surface such that it is zero when the initial time. In this manner, the sliding mode exists from the initial moment, which eliminates the phase reached the surface. Thus, the ISMC theoretically guarantees greater robustness by eliminating the static error; this is because of the modified switching surface using the integral action. However, in the presence of large uncertainties, ISMC control approach produces oscillatory phenomenon due to the higher needed gain.

This paper presents a robust rotor speed tracking controller allowing VSWT to capture maximum wind energy. The designed method is a combination of Integral action and SMC control. Due to the integral action introduced into the sliding surface, the ISMC guarantees greater robustness by eliminating the static error and by improving the performances of the controlled system in term of reaching phase and tracking error level. A comparison between standard SMC and ISMC controllers has been investigated using one mass WT model. The stability of the proposed ISMC controller was shown by the Lyapunov theory, and the simulation results were provided to demonstrate the performances of the proposed approach.

This study is organized as follows. The next section presents the VSWT modelling. Section 3 shows the design of the proposed integral sliding mode control. In
Section 4, simulation results are provided to demonstrate the robust control performance of the proposed ISMC. Finally, in section 5 a concluding remark is given.

\section{Variable speed wind turbine modeling}

\subsection{Aerodynamic torque}

Wind energy across a surface $S$ depends on the cube of the wind speed $v$, and the density of the air $\rho$. This available power is given by:

$$
P_{v}=\frac{1}{2} S \rho v^{3}
$$

where:

$$
S=\pi R^{2}
$$

$R$ is the radius of the rotor.

A variable speed wind turbine is composed of aeroturbine, a gearbox, and a generator. The aerodynamic power extracted by the rotor is given by the following equation:

$$
P_{a}=\frac{1}{2} \rho \pi R^{2} C_{p}(\lambda, \beta) v^{3}
$$

where $C_{p}$ is the power coefficient that describes the capacity of the turbine to capture energy from wind. It depends on the blade pitch angle $\beta$ and the tip-speed ratio $\lambda$ defined by:

$$
\lambda=\frac{\omega_{r} R}{v}
$$

with $\omega_{r}$ is the rotor speed.

This coefficient can be expressed by a nonlinear function of $\lambda$ and $\beta$ [12]:

$$
C_{p}(\lambda, \beta)=C_{1}\left(\frac{C_{2}}{\lambda_{i}}-C_{3} \beta-C_{4}\right) \exp \left(\frac{-C_{5}}{\lambda_{i}}\right)
$$

where

$$
\frac{1}{\lambda_{i}}=\frac{1}{\lambda+0.08 \beta}-\frac{0.035}{\beta^{3}+1}
$$

and $C_{1}$ to $C_{5}$ are a known wind turbine power coefficients.

Generally, the maximum value of $C_{p}$ for modern turbines is less than 0.5. For our study, $C_{p}$ characteristics are shown in Fig. 1 for different values of $\beta$. We noticed higher values of $C_{p}$ for small $\beta$; the turbine is most efficient for a particular $\lambda$.

By using the relationship:

$$
P_{a}=\omega_{r} T_{a}
$$

Thus, the aerodynamic torque expression is: 


$$
T_{a}=\frac{1}{2} \rho \pi R^{3} C_{q}(\lambda, \beta) v^{2}
$$

where:

$$
C_{q}(\lambda, \beta)=\frac{C_{p}(\lambda, \beta)}{\lambda}
$$

$C_{q}(\lambda, \beta)$ is the torque coefficient.

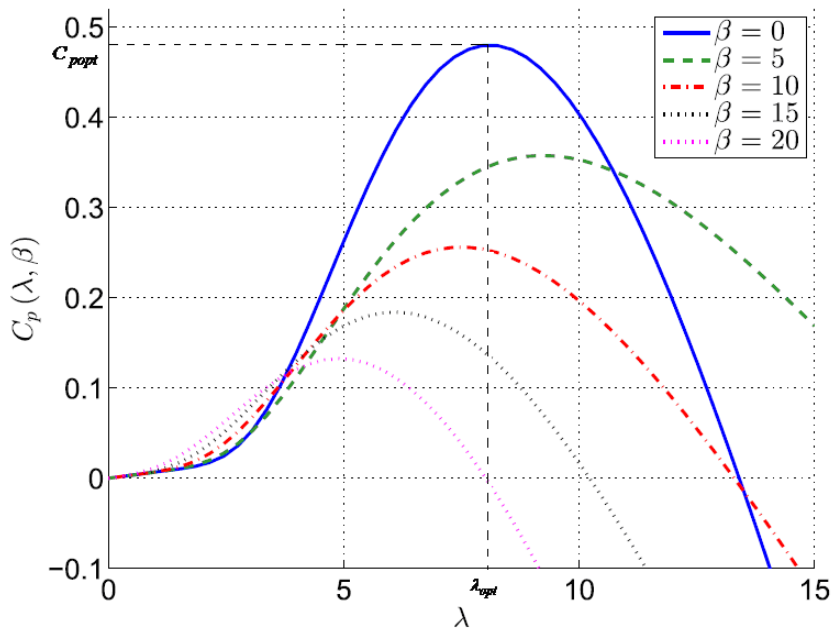

Fig. 1. WT power coefficient versus the tip-speed ratio $\lambda$.

\subsection{Large wind turbine model}

In the literature, the turbine is presented by the modelling of the mechanical part [13], or by that of the electrical part [14]. In this paper, we are interested in the modelling of the mechanical part of a large wind turbine. This last is presented by the one mass model presenting a special case of the two mass model as shown in Fig. 2.

The rotor dynamics is characterized by a differential equation of the first order:

$$
J_{r} \dot{\omega}_{r}=T_{a}-T_{l s}-K_{r} \omega_{r}
$$

$J_{r}$ and $K_{r}$ are respectively the rotor inertia and the rotor external damping.

The low-speed shaft torque $T_{l s}$ resulting effects of friction and torque generated by the differences between the rotor angular velocity $\omega_{r}$ and that of the output shaft $\omega_{l s}$ (see Fig. 2):

$$
T_{l s}=B_{l s}\left(\theta_{r}-\theta_{l s}\right)+K_{l s}\left(\omega_{r}-\omega_{l s}\right)
$$

$B_{l s}$ and $K_{l s}$ are respectively the shaft stiffness coefficient and the shaft damping coefficient.

The relationship between the high-speed shaft torque $T_{h s}$ and the generator electromagnetic torque $T_{e m}$ is given by:

$$
J_{g} \dot{\omega}_{g}=T_{h s}-K_{g} \omega_{g}-T_{e m}
$$

$J_{g}, \omega_{g}$ and $K_{g}$ are respectively the generator inertia, the generator speed and the generator friction coefficient.
Let's assume an ideal gearbox with transmission ratio $n_{g}$, we can get:

$$
n_{g}=\frac{T_{l s}}{T_{h s}}=\frac{\omega_{g}}{\omega_{l s}}=\frac{\theta_{g}}{\theta_{l s}}
$$

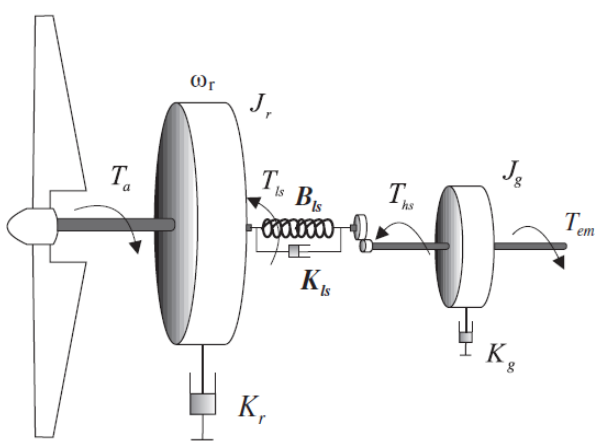

Fig. 2. Two-mass model of a wind turbine.

Transferring the generator dynamics to the low-speed side and using (12) and (13), the generator dynamics can be written as:

$$
n_{g}^{2} J_{g} \dot{\omega}_{r}=T_{l s}-n_{g}^{2} K_{g} \omega_{r}-n_{g} T_{e m}
$$

If a perfectly rigid low-speed shaft is assumed, a onemass model of the wind turbine may then be considered (Fig. 3):

$$
J_{t} \dot{\omega}_{r}=T_{a}-K_{t} \omega_{r}-T_{g}
$$

where:

$$
J_{t}=J_{r}+n_{g}^{2} J_{g}, K_{t}=K_{r}+n_{g}^{2} K_{g} \text {, and } T_{g}=n_{g} T_{e m}
$$

$J_{t}, K_{t}, T_{g}$ are respectively the turbine total inertia, the turbine total external damping and the new command.

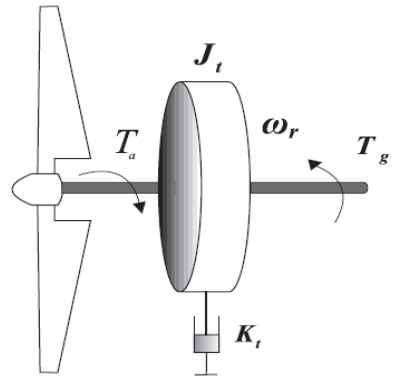

Fig. 3. One-mass model of a wind turbine.

\section{Controller Design}

The main control objective is to maximize the wind energy capture and minimize the efforts of drive train shafts. The power coefficient curve $C_{p}(\lambda, \beta)$ has a unique maximum which corresponds to the optimal wind energy:

$$
C_{p}\left(\lambda_{o p t}, \beta_{o p t}\right)=C_{p_{o p t}}
$$

The rotor, thus, provides maximum aerodynamic power only to the tip-speed $\lambda_{\text {opt }}$ :

$$
\lambda_{\text {opt }}=\frac{\omega_{\text {ropt }} R}{v}
$$

To maximize the captured energy of the wind, the variables $\lambda$ and $\beta$ must be maintained at their 
optimal values in order to ensure maximum value of $C_{p}$. So, the blade pitch angle is fixed at its optimal value $\beta_{\text {opt }}$. The tip-speed $\lambda$ depends on both of the wind speed $v$ and the rotor speed $\omega_{r}$. As the wind speed is not a controllable input, the rotor speed $\omega_{r}$ has to be regulated to track the optimal rotor speed given by:

$$
\omega_{\text {ropt }}=\frac{\lambda_{\text {opt }}}{R} v
$$

Before the controller design, let define $x=\omega_{r}, u=T_{g}$ and the system dynamic described by (6) can be rewritten as follows:

$$
\dot{x}(t)=f(x)+g u(t)
$$

where $g=\frac{-1}{J_{t}}$ and $f(x)=\frac{1}{J_{t}}\left(T_{a}-K_{t} \omega_{r}\right)$

\subsection{Sliding mode control (SMC)}

SMC is one of the most important robust control approaches against system dynamics and uncertainties $[7,8]$. Lyapunov approach is used in SMC to guarantee the stability of the nonlinear system under control. In the following, the tracking error is defined as:

$$
e=x-\omega_{\text {ropt }}
$$

Generally, two main steps are considered to design the $\mathrm{SMC}$; first finding the sliding surface and second developing the control law. Thus, the sliding surface can be expressed as:

$$
\sigma(t)=\left(\frac{d}{d t}+\gamma\right)^{r-1} e(t)
$$

where $r$ is the order of the sliding surface and $\gamma$ is a positive constant.

Since we deal with a first order system $r=1$, then, the sliding surface can be defined as:

$$
\sigma(t)=e(t)
$$

Differentiating $\sigma$ with respect to time, we can get:

$$
\dot{\sigma}=\dot{e}=f(x)+g u(t)-\dot{\omega}_{\text {ropt }}
$$

To guarantee the existence of sliding mode on a switching surface in finite time, the condition given below must be satisfied [7]:

$$
S \dot{S}<-\eta|S|
$$

where $\eta$ is a small positive constant.

The control law that satisfies Eq. (24) is given by:

$$
u(t)=-\frac{f(x)-\dot{\omega}_{\text {ropt }}}{g}-\frac{k}{g} \operatorname{sign}(\sigma)
$$

with $k$ is a positive switching gain that compensate the uncertainties and $\operatorname{sign}($.$) is the sign function defined by :$

$$
\operatorname{sign}(\sigma)= \begin{cases}1 & \text { if } \sigma>0 \\ 0 & \text { if } \sigma=0 \\ -1 & \text { if } \sigma<0\end{cases}
$$

To reduce the chattering phenomenon produce by the discontinuous control law, the boundary layer approach can be applied. Thus, the control law becomes as follows:

$$
u(t)=-\frac{f(x)-\dot{\omega}_{\text {ropt }}}{g}-\frac{k}{g} \operatorname{sat}(\sigma)
$$

where $\operatorname{sat}($.$) is the saturation function, given by:$

$$
\operatorname{sat}(\sigma)= \begin{cases}\sigma / L & \text { if }|\sigma|<L \\ \operatorname{sign}(\sigma) & \text { otherwise }\end{cases}
$$

with $L$ is the thickness of the boundary layer.

\subsection{Integral sliding mode control (ISMC)}

The response of the SMC remains slow and the chattering behavior may occur due to constant high gain. To improve transient response with minimum steady state error, the integral action can be included in the sliding surface, which makes it possible to design a sliding surface such that it is zero from the initial time [15].

The new sliding surface is defined as:

$$
\sigma(t)=\left(\gamma+\frac{d}{d t}\right)^{r-1} e(t)+k_{i} \int_{0}^{\infty} e(t) d t
$$

where $k_{i}$ is the integral gain.

The order of the system is $r=1$, then the sliding surface can be modified as:

$$
\sigma(t)=e(t)+k_{i} \int_{0}^{\infty} e(t) d t
$$

The derivative of Eq. (28) gives:

$$
\dot{\sigma}(t)=\dot{e}(t)+k_{i} e(t)
$$

Finally the torque control structure is given as:

$$
u(t)=-\frac{f(x)-\dot{\omega}_{\text {ropt }}}{g}-\frac{k_{i}}{g} e(t)-\frac{k}{g} \operatorname{sat}(\sigma)
$$

Theorem: Considering the WT system given by (19). If the control law is designed according to (34), the controlled system achieves a robust maximum power tracking. Moreover, the trajectory tracking error will converge in finite time to zero.

Proof: To demonstrate the system stability, a positive definite Lyapunov function can be introduced in the brief such as:

$$
V=\frac{1}{2} \sigma^{2}
$$

By differentiating (35) with respect to time, we can get: 


$$
\dot{V}=\sigma \dot{\sigma}=\sigma\left(f(x)+g u(t)-\dot{\omega}_{\text {ropt }}+k_{i} e(t)\right)
$$

By replacing the expression of $u$ given in (19), we have:

$$
\begin{aligned}
\dot{V} & =\sigma \dot{\sigma}=\sigma[-k \operatorname{sat}(\sigma)] \\
& =-\operatorname{kosat}(\sigma)
\end{aligned}
$$

For any $L>0, \quad$ if $|\sigma|>L, \quad \operatorname{sat}(\sigma)=\operatorname{sign}(\sigma)$ the function $\dot{V}=-k|\sigma|$. Nevertheless, in a small $L$-vicinty of the origin [7], (boundary layer), $\operatorname{sat}(\sigma)=\frac{\sigma}{L}$ is continuous, the system trajectories are limited to a boundary layer of sliding mode manifold $\sigma=0$ and $\dot{V}=-k \frac{\sigma^{2}}{L}$.

\section{Simulations results}

To highlight the performance of the proposed controller, different simulations are considered in the presence of a wind speed profile that shown in Figure 4. The one-mass model parameters are presented in Table 1. In order to make a comparison between the proposed ISMC and traditional SMC controllers, different simulations were investigated for a singlemass model wind turbine using the same conditions. For that reason, the same switching gains $(k=0.01$ and $k=0.1)$ were considered in the control law, for both controllers (i.e. ISMC and SMC).

Fig. 5 presents the reference, the real rotor speed obtained with the proposed ISMC controller and the real rotor speed obtained using a SMC controller with the same gains $(k=0.01)$. From this figure it is clear that the SMC is not able and totally failed to track the reference rotor speed. In addition, it can be seen that, the best tracking performance is obtained when the proposed ISMC control is applied. The corresponding torque input signals, presented in Fig. 6, provide a smoothness control signal without any chattering behavior was noticed. The optimal and actual aerodynamic power extracted by the wind turbine are presented in Fig. 7, from the results, it is clear that ISMC gives the best and fast tracking performance.

Fig. 8 shows the rotor speed comparison for ISMC and SMC controller in the case when $k=0.1$. From these figure, we noticed that the desired rotor speed, with the proposed controller (ISMC) was tracked satisfactorily, compared to the other method. The corresponding control signals, illustrated in Fig. 9, show the time evolution of the torque input which presents a smoothness input signal without any chattering behavior. Fig. 10 shows the time evolution of the optimal and actual aerodynamic power. As expected, the proposed ISMC controller presents a better optimization of the captured power by the turbine, due its high robustness against wind speed variations and system uncertainties. Therefore, the proposed controller provides better tracking for the optimal rotor speed command, and then a better power extraction from the wind compared to the other method.

Table 1. One-mass model parameters

\begin{tabular}{|c|c|}
\hline Parameters & Meaning \\
\hline$R=21.65 \mathrm{~m}$ & Rotor radius \\
\hline$\rho=1.29 \mathrm{~kg} / \mathrm{m}^{3}$ & Air density \\
\hline$J_{t}=3.9210^{5} \mathrm{~kg} / \mathrm{m}^{2}$ & Turbine total inertia \\
\hline$K_{t}=400 \mathrm{Nm} / \mathrm{rad} / \mathrm{s}$ & $\begin{array}{c}\text { Turbine total external } \\
\text { damping }\end{array}$ \\
\hline$\lambda_{\text {opt }}=8.26$ & the optimal tip-speed \\
\hline
\end{tabular}

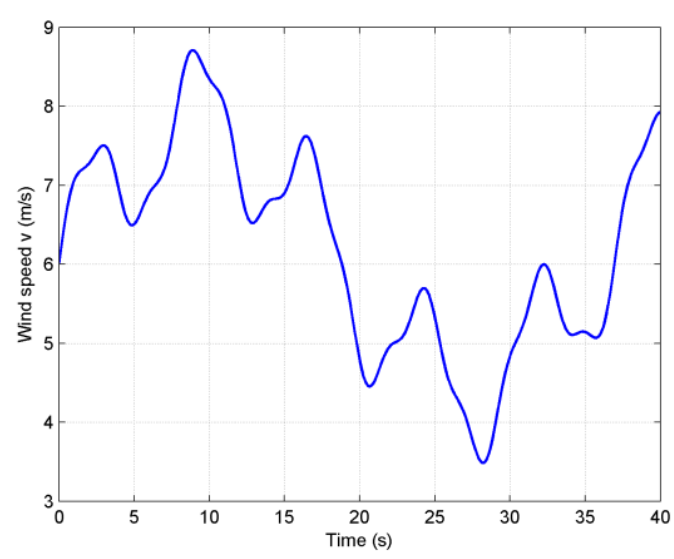

Fig.4.Wind speed profile

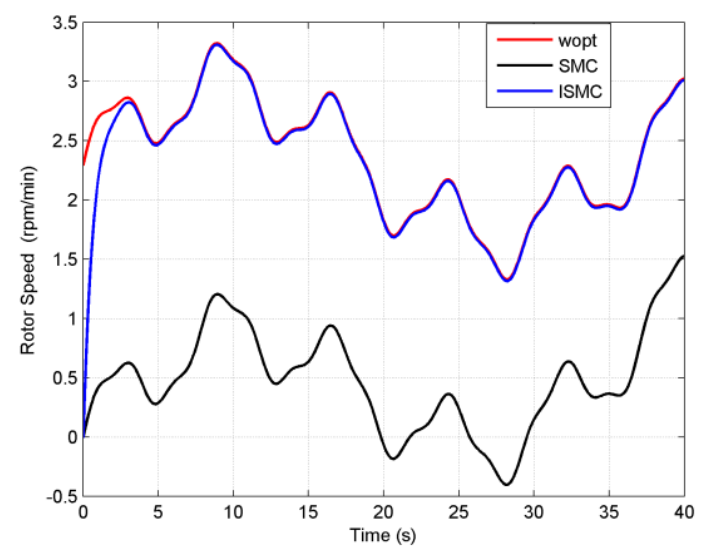

Fig. 5. Optimal and real rotor speed for $k=0.01$

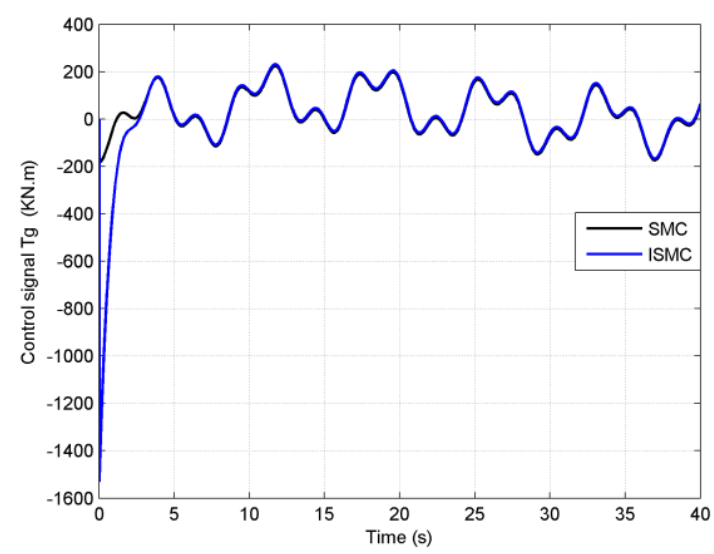

Fig. 6. Time evolution of applied torque for $k=0.01$ 


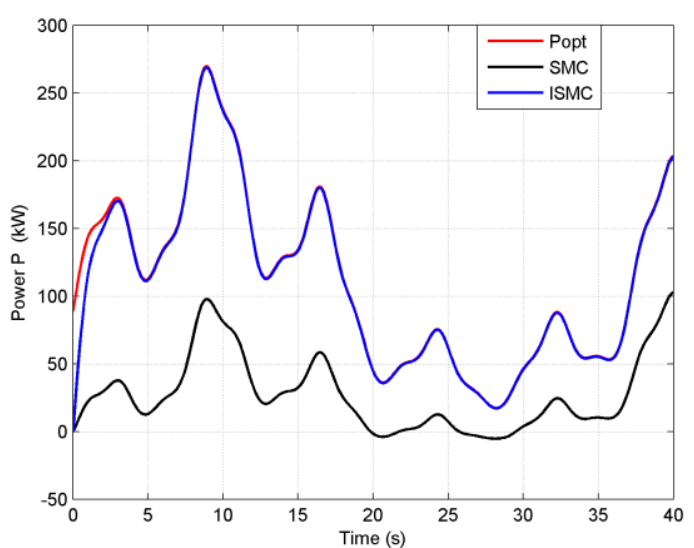

Fig. 7. Time evolution of aerodynamic power for $k=0.01$

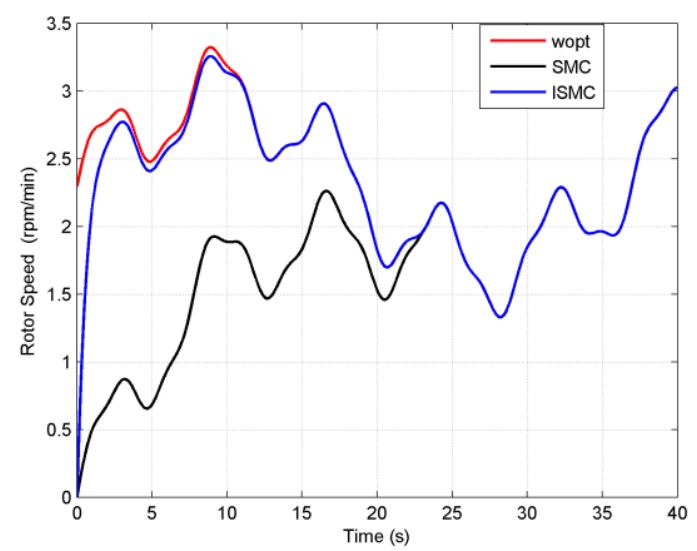

Fig. 8 Optimal and real rotor speed for $k=0.1$

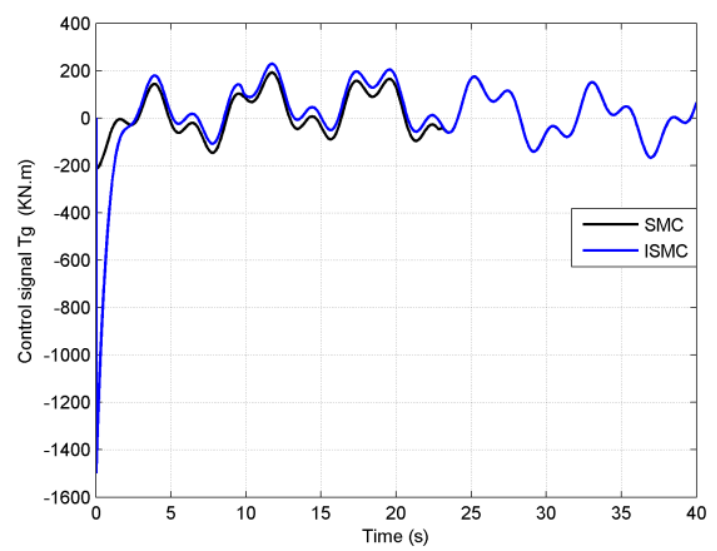

Fig. 9. Time evolution of applied torque for $k=0.1$

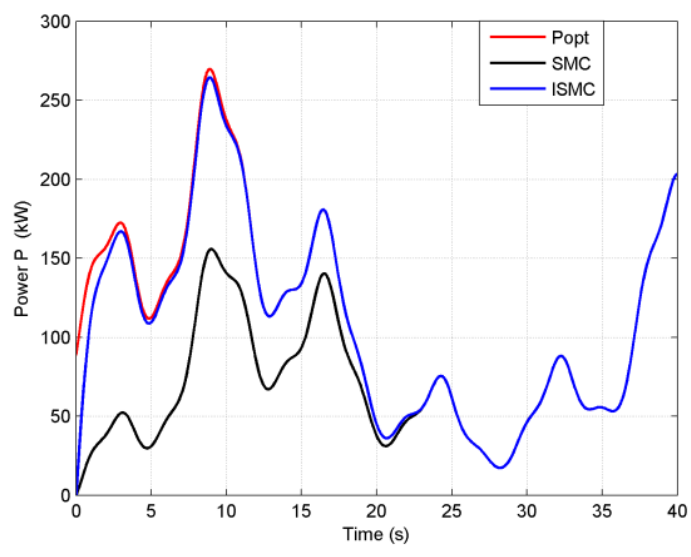

Fig. 10. Time evolution of aerodynamic power for $k=0.1$

\section{Conclusion}

This paper addressed the robust optimal reference tracking problem for a variable speed wind turbine, in order to maximize the power extracted of the wind. The designed method is a nonlinear controller based on sliding mode controller as robust method to deal with the system nonlinear behaviour. Furthermore, an integral action was introduced to the switching variable in order to improve the conventional SMC controller performance and that by reducing the transition response time and skipping the reaching phase. The comparison with the traditional sliding mode control has been realized and simulation results illustrate the effectiveness of the controller, and show that the proposed approach has provided, compared to other method, a faster transit response. Further studies will report on more effective and efficient control approaches for the entire energy system, including the electrical part. An experimental validation of the presented results will be also a subject of our future work.

\section{References}

[1] J.F. Manwell, J.G. McGowan, and A.L. Rogers, Wind energy explained: theory, design and applications , John Wiley \& Sons (2002)

[2] G. Ofualagba and E. U. Ubeku, Wind energy conversion system- wind turbine modelling, Power and Energy Society General Meeting Conversion and Delivery of Electrical Energy in the 21st Century, IEEE, 1-8 (2008)

[3] T. Burton, D. Sharpe, N. Jenkins, and E. Bossanyi, Wind energy handbook, John Wiley \& Sons (2001)

[4] S. Wang, S. Habibi, and R. Burton, The Smooth Sliding Mode Controller and Filter, Control and Intelligent Systems 38(3), 130-139 (2010)

[5] P.F. Puleston and F. Valenciaga, Chattering reduction in a geometric sliding mode method. A robust low-chattering controller for an autonomous wind system, Control and Intelligent Systems 37(1), 39-45 (2009) 
[6] H. Amimeur, D. Aouzellag, R. Abdessemed, and K. Ghedamsi, Sliding mode control of a dual-stator induction generator for wind energy conversion systems, International Journal of Electrical Power and Energy Systems 42(1), 60-70 (2012)

[7] V.I. Utkin, Sliding modes in control optimization, Springer-Verlag (1992)

[8] J.J. Slotine, Sliding controller design for nonlinear systems, International Journal of Control 40(2), 421-434 (1984)

[9] D. Jena and S. Rajendran, A review of estimation of effective wind speed based on control of wind turbines, Renew Sust Energy Rev 43, 1046-1052 (2015)

[10] S. Rajendran and D. Jena, Validation of an integral sliding mode control for optimal control of a three blade variable speed variable pitch wind turbine, Electrical Power and Energy Systems 69, 421-429 (2015)

[11] S. Li, H. Wang, T.Tian, A. Aitouch and J. Klein, Direct power control of DFIG wind turbine systems based on an intelligent proportional-integral sliding mode control, ISA Transactions 64, 431-439 (2016)

[12]H.J. Asl and J. Yoon, Power capture optimization of variable-speed wind turbines using an output feedback controller, Renewable Energy 86, 517-525 (2016)

[13] J.M. Rubio and LT. Aguilar, Maximizing the performance of variable speed wind turbine with nonlinear output feedback control, Procedia Engineering 35, 31-40 (2012)

[14] Ph. Delarue, A. Bouscayrol, A. Tounzi, X. Guillaud, and G. Lancigu, Modelling, control and simulation of an overall wind energy conversion system, Renewable Energy 28(8), 1169-1185 (2003)

[15] V. Utkin, and J. Shi, Integral sliding mode in systems operating under uncertainty conditions, Digests 35th Annual Conf. IEEE on Dicision and Control, 4591-4596 (1996) 